\title{
GATHERING RESEARCHERS' REQUIREMENTS TO DEVELOP A LEARNING TECHNOLOGIES DASHBOARD
}

\author{
Miguel Alonso Canizares Mena and Pedro Teixeira Isaias \\ The University of Queensland \\ St Lucia, Queensland - Australia
}

\begin{abstract}
The learning technologies area has expanded incredibly fast due to the growing and acceptance of technology in the classrooms. As a result, the data produced by students is vast and is growing in each of the learning technologies used, leading to a possibility of discovering patterns that can help to enhance the student experience. However, a clear research regarding the adoption of learning technologies and how to spread this research is limited and almost inexistent. Most of the research revolves around students or teachers disregarding the researcher's standpoint. All the tools and artefacts such as dashboards that have helped to improve both students and teachers' knowledge are absent for researchers. Hence, this paper is aimed on finding and defining the most common learning technologies platforms and tools as well as the user requirements to build a dashboard to display this information considering the researchers' objectives. In this regard, an online survey was created and sent to both researchers interested in the learning technology area and learning technologies designers. The responses were analysed with quantitative tools such as exploratory factor analysis, analysis of variance, t-tests and graphical means. The results led to the user requirements for developing a dashboard.
\end{abstract}

\section{KEYWORDS}

Learning Technologies, Learning Analytics, Dashboard Design, Quantitative Research

\section{INTRODUCTION}

The massive consumption of technology is a growing area as new generations are more connected to technology than ever before. Resources, such as laptops and mobile phones, are available to them from an early age (Glenn, 2008). Devices and connectivity are entering every aspect of human life, including education. During the latest boom, technology has brought huge changes to the learning methodology used in classrooms. These changes affect students, teachers, institutions and researchers - almost every actor in the learning environment (Bothma \& Cant, 2011). The inclusion of technology has changed the traditional pedagogy used in educational institutions and modified the centre of learning, moving from a teacher-centred classroom to a student-centred classroom (Ariso, 2017).

The tools created specifically for the learning environment to enable active pedagogy methodologies are called 'learning technologies'. These are technologies created to improve, enhance and support the student's learning (Aleven, Beal \& Graesser, 2013).

Information and communication technology (ICT), is the technical section of the tools that involves the programming of the software that is going to be used in the classroom. The ICT enables humans to achieve goals that would have been impossible to accomplish before technology (Cerri, 2012). Although the rapid development of technology and internet is one of its great qualities, it also poses one of its great challenges.

The psychological section mainly involves the pedagogy behind the development and use of specific learning technology characteristics, such as student's motivation, enjoyment, engagement, expectations and values (Jackson \& McNamara, 2013). One of the major changes to the traditional pedagogy is its transformation from a passive to an active method. This encourages teachers to develop more practical and challenging activities, rather than the traditional lecture format (Paquette, Randall-Schab \& Busacca, 2015). 
The introduction of learning technologies tools and platforms have increased interest in other research areas, such as learning analytics. Learning analytics have come to the forefront of researchers' focus as these tools are enablers of techniques and are a source of data for future studies.

While the learning analytics tools and frameworks are still evolving, one of the biggest challenges of analytics techniques is how to report the findings. There is more information than ever, which becomes more complex to analyse, leading to the use of more complicated analytics techniques. The raw outcomes can overwhelm a person. To tackle this limitation, visual analytics is used to report statistical outcomes. This provides clean and understandable results that are easy to communicate to stakeholders (Keim et al., 2008). Within the visual analytics framework, one of the leading tools used to report information is the dashboard.

One of the biggest challenges of creating a dashboard is finding the correct requirements of the users and the purpose of the tool (Few, 2007). This is considerably difficult as sometimes not even the final users know exactly what they need and so what they want can be misunderstood. The most useful techniques to tackle this issue are surveys and interviews (Bethlehem, 2009). Even once the requirements are established, there is still the design of the dashboard that must be considered. The presentation of the dashboard is important as it can amplify human cognition, performance and support (Card, Mackinlay \& Shneiderman, 1999).

As stated previously, the learning technologies and analytics fields are new, being active for less than a decade. Additionally, research so far is spread around the world with no commonality. Although some papers have begun to analyse and compile previous research to present the path that the area is taking, there is still a lack of tools to share this information with the wider research community. Papers such as those by Schwendimann et al. (2017) and Verbert, Duval, Klerkx, Govaerts and Santos (2013) have marked the trend to present the results and the current state of the research. Consequently, there is a need among learning technologies researchers to receive current updates on the state of the research to provide them with the opportunity to drill down in the information and find what has been done and how it can be implemented.

This paper outlines the process of finding research on the current state of learning technologies and dashboard design. The literature review was used to determine what the most important learning technologies around the world are and which platforms are the most popular.

\section{LITERATURE REVIEW}

\subsection{Learning Technologies}

Learning technologies are defined as technologies that support and improve teaching and/or learning that may include virtual platforms or learning environments (Kumar \& Daniel, 2016; Sife, Lwoga \& Sanga, 2007). Learning technologies is broad and can include hardware, software and online applications. The inclusion of technology in classrooms has increased in the last decade for two main reasons: positive learning outcomes and low cost. Research has demonstrated that the use of learning technologies can increase the quality of learning and the student experience (Karasavvidis \& Kollias, 2014) and the ease of access to powerful computational devices at the cheapest price (Zoya \& Valeriy, 2016). According to Drent and Meelissen (2008), students can benefit from learning technologies in developing skills such as collaboration, communication and problem-solving. Teachers can improve their pedagogy, resulting in a better quality of teaching (Levin \& Wadmany, 2008; Mends-Brew, 2012). The newest devices are becoming smaller and more powerful, leading to a tendency that has mobility and reachability as main characteristics (BenMoussa, 2003; Camponovo \& Pigneur, 2003; Ng-Kruelle, Swatman, Rebne \& Hampe, 2002). This has resulted in efficiency and effectivity in teaching and learning (Maginnis, White \& Mckenna, 2000). All of the characteristics of learning technologies have led to its rapid development (Fernández-Ferrer \& Cano, 2016). As such, there is disorganisation as a consequence of learning technologies being created by different sources. Some literature has defined the different tools available within the learning technologies framework: learning management systems, mobile learning tools, social networks, video conferencing, collaboration tools, gamification tools, active-learning tools, virtual augmented reality platforms and MOOCs (Bothma \& Cant, 2011; Harley, Lajoie, Frasson \& Hall, 2017; Kasapoglu-Akyol, 2010). 


\subsection{Dashboard Design}

A dashboard is defined as a visual interface that provides critical information in the form of visualisations for stakeholders (Wolf, 2016). It can also be added that the information provided must be in real-time (Wexler, Shaffer \& Cotgreave, 2017). The idea of a dashboard is to simplify critical information into a single screen, providing an overview of the data (Key et al., 2012). There are researchers who argue that a dashboard leaves out important data in its objective to simplify the information. Opponents to dashboard argue that simplifying the information leads to problems such as misaligned, incorrect and imposed indicators (Allio, 2012). The premise of the opponents is that 'complex scenarios cannot be simplified into few indicators' (Allio, 2012). Supporters of dashboard argue that providing few critical indicators in a clear and concise form results in an increase in the human cognition and processing of information (Card et al., 1999). This is supported by several human cognition studies that demonstrate that a visual material is easier for the human brain to process (Ware, 2004) due to its inherent analytical and intuitive qualities (Patterson, Pierce, Bell, Andrews \& Winterbottom, 2009). The human brain is skilled in perceiving patterns, the relationship between different domains, deduction and logical conclusions and storing information in the long-term section of the brain (Patterson et al., 2014). According to Patterson, Pierce, Bell and Klein (2010), the combination of these characteristics leads to better decision-making due to the robust activities performed by the human brain while processing this information. To create effective visualisations, the human cognition literature recommends focusing on a three-stage hierarchical framework (Ware, 2004). The first stage is a lower stage that processes basic elements of the visualisation, such as shape, colour and texture. The second stage is a middle stage that processes complex information within objects such as patterns and motion. The third stage is a higher stage that processes relations between the objects (Patterson et al., 2014). To create effective visuals to be included later in dashboards, guidelines have been created by some researchers (Fisher, Green \& Arias-Hernandez, 2011; Thomas \& Cook, 2006). According to Patterson et al. (2014), six leverage points can be defined that will increase the efficiency of visualisations; the first three are regarding the lower and middle-stage processes in which information about the object is processed. The final three considers the relationships of the objects or decision-making.

\section{MODEL AND HIPOTHESIS}

The research method selected to develop this thesis is conceptual design science research (CDSR) due to the limitation of the empirical testing of the artefact created. This research method was described by Gleasure (2014) as the foundation for research that develops an artefact based on theory or user requirements.

Gleasure (2014) proposes the following guidelines for developing CDSR.

First, the behavioural outcomes of the design must be identified. The behavioural outcomes will act as meta-requirements for guiding the design. The purpose of meta-requirements is to provide a broad specification in terms of scale and scope of the user's needs (Ceccarelli, Mori, Lollini \& Bondavalli, 2015).

Second, the existing artefacts with a similar set of behavioural outcomes must be identified. The platforms were identified using the paper by Schwendimann et al. (2017). In this paper, the platforms that were selected were those in which the target users included researchers.

Third, the key differentiating design features must be analysed in each artefact. The main differences that were found were the platform used to provide access to the artefacts, the tools used to develop the artefacts in specific data collection and the grouping and interactivity of the visualisations. Table 1 provides a clear picture of the relationship between the behavioural outcomes and the design features.

Table 1. Matrix Relationship

\begin{tabular}{lllllll}
\hline Existing & Data & Visualizations & Data & Access & Data & Visualizations \\
Artefacts & Integration & Grouping & Processing & & Collection & Interactivity \\
\hline EngAGe & High & High & High & Web & APIs & Medium \\
TFM & Medium & Medium & High & Web & APIs & Low \\
moocRP & Low & Low & Medium & App & Manual & High \\
eMUSE & High & Medium & Medium & Web & APIs & Low \\
SCALE & Medium & Low & High & Web & APIs & Low \\
\hline
\end{tabular}




\section{METHODOLOGY}

To build an effective information system, it is essential to find the correct user requirements (Nuseibeh $\&$ Easterbrook, 2000). Although the requirements are usually focused on the user, other factors - such as the technical, cultural and social environments - must be considered to develop an information system (Sawyer \& Kotonya, 2001). While these factors must be studied, only the user requirements involve a human factor. Thus, these are the ones that require deeper research. Sommerville (2007) found three main difficulties while working with humans: users usually do not know exactly what they need, the language they use to describe the requirements is often too related to the domain and different users may have different requirements. To tackle these limitations, this paper designed a questionnaire that comprehensively analysed the literature in the learning technologies area along with existing surveys that found the popularity of each platform in the education industry that might be of interest for researchers. Table 2 describes the principal learning technologies and platforms discovered in the literature review and its respective popularity (Hart, 2018).

Table 2. Learning Technologies Global Popularity

\begin{tabular}{llll}
\hline Group Name & Learning technology & Group Popularity & Global popularity \\
\hline LMS & Moodle & 1 & 16 \\
& Google classroom & 3 & 54 \\
& Blackboard & 4 & 55 \\
Social network platform & Edmodo & 9 & 85 \\
& YouTube & 1 & 2 \\
& Wikipedia & 2 & 13 \\
& Twitter & 3 & 15 \\
Video conferencing & Facebook & 4 & 17 \\
& Zoom & 2 & 10 \\
& Skype & 3 & 26 \\
Collaboration tools & Adobe Connect & 4 & 28 \\
& Google Hangouts & 6 & 50 \\
& Padlet & 1 & 7 \\
Active learning tools & Slack & 2 & 31 \\
& G Suite & 5 & 40 \\
& Microsoft SharePoint & 10 & 101 \\
& Kahoot! & 1 & 6 \\
MOOCs & Quizlet & 3 & 23 \\
& Pool Everywhere & 4 & 29 \\
& Socrative & 7 & 56 \\
Mobile learning tools & Coursera & 6 & 101 \\
Gamification tools & edX & 8 & 101 \\
Virtual/augmented reality tools & All & 10 & 101 \\
\hline & Adacity & 11 & 101 \\
& Future Learn & N/A & N/A \\
& All & N/A & N/A \\
\hline
\end{tabular}

Despite the extensive research done to gather information of all the learning technologies specified, a popularity index for technologies such as mobile learning tools, gamification tools, and virtual/augmented reality was not found. Therefore, the selection of the technologies was based only on existing literature.

In order to find the constructs needed for the development of the dashboard, it was taken the design criteria stated by Marx et al (2011). Table 3 shows the constructs and its respective design criteria.

Table 3. Constructs and Questionnaire Design Criteria

\begin{tabular}{ll}
\hline Construct & Design Criteria \\
\hline Learning technologies & Scope of information \\
Individual platforms in each learning technology & Scope of information \\
Dashboard visualizations & User interface \\
Dashboard aggregations & System functions \\
Dashboard maintenance & Information management \\
\hline
\end{tabular}




\section{DATA COLLECTION}

The foundation for this project is a thorough literature review, which will provide the first learning technologies insights. The main characteristics were obtained, in addition to the most frequently used tools in classrooms worldwide. This information was transformed into an online survey following the criteria by Marx et al. (2011), using the platform, Qualtrics, and was sent via email to a testing group composed of eight people. Four participants were related to the information systems field and four were not. The purpose of the testing was to provide insights regarding the construction of the survey, including poorly structured questions or if there was more information required to complete the questionnaire successfully and to consider respondent fatigue. After one week of being deployed, the survey gathering process was stopped and six answers were received, providing the following feedback: most of the participants agreed that more information was required to complete the questionnaire. Terms such as learning technologies and platforms were unknown. The level of fatigue described by participants was described as low. Half of the participants agreed that the instructions or the platform did not provide clear guidance for answering the questions. The default ranking order was not accepted as an answer by the platform. One participant noted that the demographic question about gender should be more inclusive and allow a third option for people who identify as non-binary. Another respondent detected several grammatical errors in some questions and in a set of instructions. To improve the survey and to address the most common issues raised by the group's participants, a website was created on a free platform called 'Wix'. The website contained a description of each learning technology as well as a description of each of the platforms proposed in the survey. The final survey was sent via email and were gathered 109 responses.

\section{ANALYSIS AND RESULTS}

Questions that were considered the most important in the statistical analysis were chosen due to the low quantity of answers obtained that limited the number of variables that could be analized. The remaining questions were analysed using graphs to show the trends of the answers. The statistical method used was an exploratory factor analysis. This was used because of the absence of models in the learning technologies area, with a focus on researchers. The statistical results given by the exploratory factor analysis were not strong enough to provide a robust fit. Although there were relationships between learning technologies and dashboard design sections that have a high correlation and covariance, the model did not reach the minimum required to be considered significant. As a consequence, an ANOVA (analysis of variance) analysis and t-tests were used to check the relationships between elements within these groups (Law, 2016).

\subsection{Analysis of variance: ANOVA}

ANOVA is a powerful tool for checking the interaction between variables or factors. ANOVA leads to the decision of whether or not to reject the null hypothesis; that is, if the variable's mean is statistically different. This analysis does not specify what variable is the one that has a statistically different mean (Smalheiser, 2017b). To discover further insights, t-tests must be performed between variables to discover what variables are statistically different. A t-test compares the means between two variables to discover if there is a statistically significant difference (Smalheiser, 2017a). To check the previous relationships found in the exploratory factor analysis, three ANOVAs were performed on the observations.

The first ANOVA addressed the learning technologies variables as, according to the exploratory factor analysis, these variables were grouped into one factor. The analysis resulted in a rejection of the null hypothesis $[\mathrm{F}=119.68>\mathrm{Fcrit}=1.88]$. To check what variable or variables have this difference a t-test was performed. The results showed that five pairs of variables were not statistically significant ( $\mathrm{p}$-value $>0.05$ ). A p-value, or reference, of 0.05 was used for all the tests as it is a standard used. Table 4 describes what pair of variables were not statistically significant and their $\mathrm{p}$-value. 
Table 4. Learning Technologies t-test Results (p-value >0.05)

\begin{tabular}{lll}
\hline Variable 1 & Variable 2 & P - Values \\
\hline Mobile learning tools & Collaboration tools & 0.630 \\
Mobile learning tools & Active learning tools & 0.183 \\
Social networks & Video conferencing tools & 0.782 \\
Collaboration tools & Active learning tools & 0.225 \\
Gamification tools & MOOCs & 0.976 \\
Virtual/augmented reality & MOOCs & 0.051 \\
\hline
\end{tabular}

The second ANOVA addresses the types of visualisation variables as, according to the exploratory factor analysis, these variables were grouped into one factor. The analysis resulted in a rejection of the null hypothesis $[\mathrm{F}=108.21>$ Fcrit $=2.39]$. To check which variable or variables have this difference a t-test was performed. The results showed that three pairs of variables were not statistically significant ( $p$-value $>0.05)$. Table 5 describes what pair of variables were not statistically significant and their p-value.

Table 5. Visualizations Type t-test Results (p-value > 0.05)

\begin{tabular}{lll}
\hline Variable 1 & Variable 2 & P - Values \\
\hline Line graphs & Pie charts & 0.753 \\
Line graphs & Network diagrams & 0.903 \\
Pie charts & Network diagrams & 0.700 \\
\hline
\end{tabular}

The third ANOVA addresses the aggregations variables as, according to the exploratory factor analysis, these variables were grouped into one factor. The analysis resulted in a rejection of the null hypothesis $[\mathrm{F}=195.33>$ Fcrit $=2.39]$. To check what variable or variables have this difference, a t-test was performed. The results showed that two pairs of variables were not statistically significant (p-value >0.05). Table 6 describes what pair of variables were not statistically significant and their p-value.

Table 6. Aggregations t-test Results (p-value > 0.05)

\begin{tabular}{lll}
\hline Variable 1 & Variable 2 & $\mathrm{P}$ - Values \\
\hline Region & Country & 0.086 \\
User & Educational institution level & 0.751 \\
\hline
\end{tabular}

To gain a better understanding of the needs of the researchers, all of the questions were analysed graphically by aggregating responses according to the level of importance and adding weights according to their ranking. Each answer was weighted with 10 points if was ranked first and with 1 point if it was ranked last. The comparison between learning technologies is shown in Figure 1.

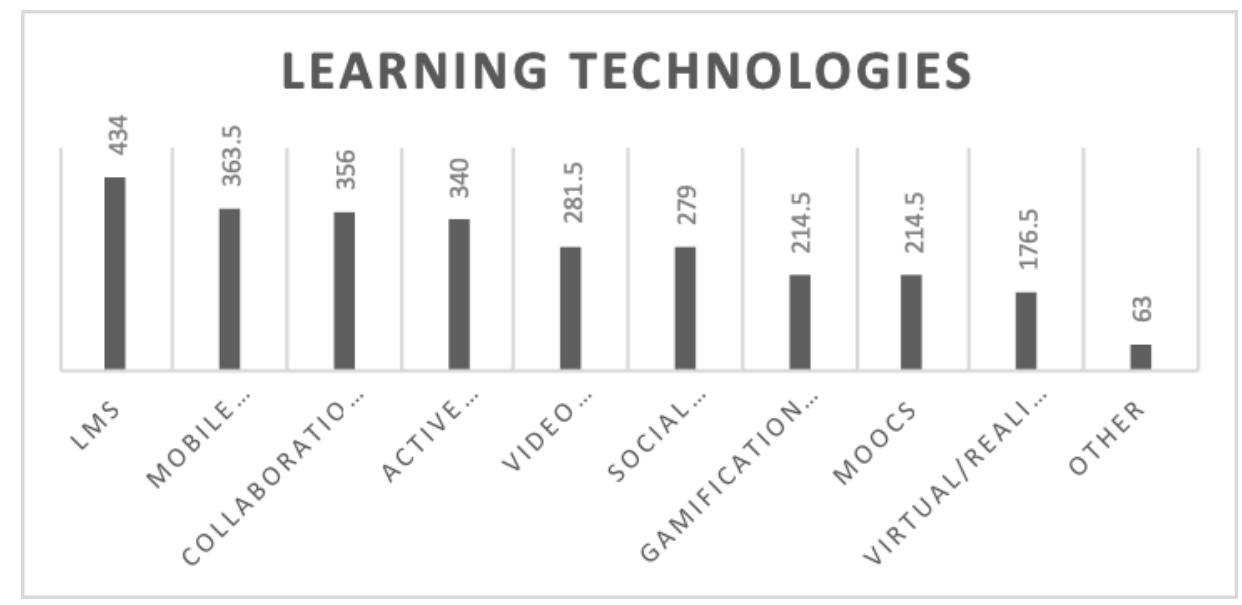

Figure 1. Weighted Learning Technologies Importance Ranking 
As shown in Figure 1, the most important learning technology for researchers is LMS. This is followed by mobile learning tools and collaboration tools. It is important to mention that, according to the results of the t-tests, mobile learning tools, collaboration tools and active-learning tools do not have a statistical difference, resulting in a possible grouping of technologies that will fall second in importance.

A comparison was conducted between learning technologies platforms or tools most used. This information is used to describe what platform or tools is considered most important in each section. According to the results, the most popular platforms were as follows: Learning management systems Moodle, Mobile learning tools - Laptops, Social network platforms - YouTube, Video conferencing technologies - Skype, Collaboration tools - Google suite, Gamification tools - Classcraft, Active learning tools - Kahoot!, MOOCs platforms - Coursera. The most popular type of visualization was bar charts and the second one pie charts. However, according to the t-tests results, there is no statistical mean difference between pie charts, line graphs, and network diagrams. Thus, for the design can be chose the second most popular option. The same occurred with the aggregations. The most popular options were active users and educational institutional level. Even though according to the t-tests there is no statistical mean difference between these two, both of them have higher values than its followers.

\section{CONCLUSION}

The learning technologies field requires further research, categorisation, a more structured way to grow and different standpoints. Almost all of the research in this area is focused on the advantages and effects of these tools on students and teachers. Due to the impressive growth of learning technologies, researchers require support and resources in which they can find meaningful information to increase the efficiency of their research. In this sense, the dashboards that, for many years, have improved decision-making in fields such as business, health and transportation, can provide support to researchers and improve decision-making before beginning a new project. One of the most difficult steps to beginning research is finding and describing the problem, in addition to finding a starting point from which to gather useful information (Alvesson \& Sandberg, 2011). To address this issue, this paper focused on providing insights about learning technologies, in addition to tools or platforms that are considered most important by researchers. Responses from the survey provide strong foundations of the characteristics required in a dashboard for researchers in the field of learning technologies. Although a clear factorial analysis was not possible due to lower KMO test results, there is evidence that a relationship exists between learning technologies and design characteristics. While the categorisation that was used was comprehensive, some similarities were found. This can reduce the amount of learning technologies to combine technologies for broader research. The t-tests results showed that some learning technologies have no statistical mean difference leading to a possible grouping reducing the learning technologies categories. In this regard the learning technologies shown in Table 4 are the ones that have a p-value higher than 0.05 , resulting in a no statistical mean difference. As a result, table 7 depicts the possible grouping and its label according to the characteristics shared among the technologies of the group.

Table 7. Learning Technologies Factors

\begin{tabular}{lll}
\hline Mobile online tools & Social network communication tools & Online presence tools \\
\hline Mobile learning tools & Social networks platforms & MOOCs \\
Collaboration tools & Video conferencing tools & Gamification tools \\
Active learning tools & & Virtual/augmented reality \\
\hline
\end{tabular}

This grouping can be helpful for further research that may focus on a specific tool to study its effects on students or teachers and provide insights regarding the current state of the research. For example, the most studied learning management system is Blackboard. However, according to the results of the survey, this platform is falling behind Moodle and Google Classroom. Additionally, the studies focusing on Google Classroom are minimal, meaning that more research must be done that focuses on Google Classroom to adequately understand the advantages and disadvantages of this platform.

The results of the dashboard design section can be used as a starting point in different research fields as these can be standardised to define researchers' requirements and to provide insights about the characteristics of a dashboard. In this regard, the results of the survey showed that the most important type of visualisation is bar charts and that there is no clear second choice for researchers. It can be concluded that a dashboard 
should at least contain a bar chart visualisation. To some extent, this result highlights the importance of bar charts in both the statistical and the graphical analysis and might indicate that this type of visualisation is the most important. It can be tested in other research areas to establish if the results are similar or not. The results of the survey indicated the need for a portal characteristic embedded in the dashboard. This is a useful insight as it can be determined that as the users of the dashboard are researchers and so, for this type of user, a descriptive information section is required.

This paper was limited by time as it was completed in a short period of time. As a consequence, the dashboard could not be tested. This would be the next step of this project, the evaluation of the prototype and further improvements to the dashboard. To proceed with the evaluation, it would be necessary to apply for an extension of the human ethics approval and submit a new questionnaire for evaluation. To accomplish the evaluation task, the best model to follow would be the technology acceptance model (TAM). Although the model is simple, it is an adequate starting point for a first evaluation. The prototype can be improved by the creation of a panel of experts to provide initial feedback about the tools and to encourage other researchers and learning technologies designers to continue to provide more feedback and information for the dashboard.

\section{REFERENCES}

Aleven, V., Beal, C. R. \& Graesser, A. C. (2013). Introduction to the special issue on advanced learning technologies. Journal of Educational Psychology, 105(4), 929-931.

Allio, M. K. (2012). Strategic dashboards: designing and deploying them to improve implementation. Strategy \& Leadership, 40(5), 24-31.

Alvesson, M. \& Sandberg, J. (2011). Generating research questions through problematization. The Academy of Management Review, 36(2), 247-271.

Ariso, J. (2017). Augmented Reality (Vol. 11). Berlin, Germany: De Gruyter.

BenMoussa, C. (2003). Workers on the move: New opportunities through mobile commerce. Paper presented at the UKAIS Conference, University of Warwick.

Bethlehem, J. (2009). Applied survey methods: A statistical perspective. Hoboken, NJ: Wiley.

Bothma, C. H. \& Cant, M. C. (2011). Adopting learning technologies: From belief to practice. Educational Studies, 37(4), 375-389.

Camponovo, G. \& Pigneur, Y. (2003). Business model analysis applied to mobile business. Paper presented at the 5th International Conference on Enterprise Information Systems (ICEIS), Angiers, France.

Card, S., Mackinlay, J. \& Shneiderman, B. (1999). Readings in information visualization: using vision to think. San Francisco, CA: Morgan Kaufmann Publishers.

Ceccarelli, A., Mori, M., Lollini, P. \& Bondavalli, A. (2015). Introducing meta-requirements for describing system of systems. Paper presented at the IEEE International Symposium on High Assurance Systems Engineering.

Cerri, S. A. (2012). Advanced learning technologies. In N. Seel (Ed.), Encyclopedia of the Sciences of Learning (pp. 154-157). New York: Springer.

Drent, M. \& Meelissen, M. (2008). Which factors obstruct or stimulate teacher educators to use ICT innovatively? Computers \& Education, 51(1), 187-199.

Fernández-Ferrer, M. \& Cano, E. (2016). The influence of the internet for pedagogical innovation: Using twitter to promote online collaborative learning. International Journal of Educational Technology in Higher Education, 13(1), $1-15$.

Few, S. (2007). Dashboard confusion revisited. (2007, March) Perceptual Edge Visual Business Intelligence Newsletter.

Fisher, B., Green, T. M. \& Arias-Hernandez, R. (2011). Visual analytics as a translational cognitive science. Topics in Cognitive Science, 3(3), 609-625.

Gleasure, R. (2014). Conceptual design science research? How and why untested meta-artifacts have a place in IS. Paper presented at the International Conference on Design Science Research in Information Systems, Karlsruhe, Germany.

Glenn, M. (2008) The Economist Intelligence Unit. The Future of higher education: How technology will shape learning [White paper]. Retrieved from http://graphics.eiu.com/upload/the\%20future\%20of\%20universities.pdf.

Harley, J. M., Lajoie, S. P., Frasson, C. \& Hall, N. C. (2017). Developing emotion-aware, advanced learning technologies: a taxonomy of approaches and features. International Journal of Artificial Intelligence in Education, $27(2), 268-297$.

Hart, J. (2018). 12th Annual Digital Learning Tools Survey. Retrieved 03/03/2019, from Centre for Learning \& Performance Technologies. 
Jackson, G. T. \& McNamara, D. S. (2013). Motivation and performance in a game-based intelligent tutoring system. Journal of Educational Psychology, 105(4), 1036-1049.

Karasavvidis, I. \& Kollias, V. (2014). Technology integration in the most favourable conditions: Findings from a professional development training program. In C. Karagiannidis, P. Politis \& I. Karasavvidis (eds) Research on e-Learning and ICT in education. Springer (pp. 197-224). New York, NY: Springer.

Kasapoglu-Akyol, P. (2010). Using educational technology tools to improve language and communication skills of ESL students. Novitas - Research on Youth and Language (ROYAL), 4(2), 225-241.

Keim, D., Andrienko, G., Fekete, J. D., Görg, C., Kohlhammer, J. \& Melançon, G. (2008). Visual analytics: Definition, process, and challenges. In A. Kerren, J. T. Stasko, J. D. Fekete \& C. North (eds.) Information Visualization. Lecture notes in computer science, Vol 4950. (pp. 154-175). Berlin, Heidelberg: Springer.

Key, A., Howe, B., Perry, D. \& Aragon, C. (2012). VizDeck: Self-organizing dashboards for visual analytics. Paper presented at the 2012 ACM SIGMOD International Conference on Management of Data, Arizona, USA.

Kumar, S. \& Daniel, B. (2016). Integration of learning technologies into teaching within Fijian polytechnic institutions. International Journal of Educational Technology in Higher Education, 13(1), 1-17.

Law, J. (2016). A Dictionary of Business and Management (6th ed.): Oxford, United Kingdom: Oxford University Press.

Levin, T. \& Wadmany, R. (2008). teachers' views on factors affecting effective integration of information technology in the classroom: Developmental scenery. Journal of Technology and Teacher Education, 16(2), 233-263.

Maginnis, F., White, R. \& Mckenna, C. (2000). Customers on the move: m-Commerce demands a business object broker approach to EAI. eAI Journal, 58-62.

Marx, F., Mayer, J. \& Winter, R. (2011). Six principles for redesigning executive information systems-findings of a survey and evaluation of a prototype. ACM Transactions on Management Information Systems (TMIS), 2(4), 1-19.

Mends-Brew, E. (2012). Enhancement of ICT in polytechnics for accelerated development. Natural and Applied Science, 3(3), 99-107.

Ng-Kruelle, G., Swatman, P., Rebne, D. \& Hampe, F. (2002). The price of convenience: Privacy and mobile commerce. Quarterly Journal of Electronic Commerce, 3(3), 273-285.

Nuseibeh, B. \& Easterbrook, S. (2000). Requirements engineering: A roadmap. Paper presented at the IEEE International Conference on Software Engineering, Limerick, Ireland.

Paquette, L., Randall-Schab, M. \& Busacca, J. (2015). Teaching political science to undergraduates: active pedagogy for the microchip mind. Warsaw/Berlin: De Gruyter Open.

Patterson, R., Blaha, L., Grinstein, G., Liggett, K., Kaveney, D., Sheldon, K. \& J. Moore. (2014). A human cognition framework for information visualization. Computers \& Graphics, 42(1), 42-58.

Patterson, R., Pierce, B., Bell, H., Andrews, D. \& Winterbottom, M. (2009). Training robust decision making in immersive environments. Journal of Cognitive Engineering and Decision Making, 3(4), 331-361.

Patterson, R., Pierce, B., Bell, H. \& Klein, G. (2010). Implicit learning, tacit knowledge, expertise development, and naturalistic decision making. Journal of Cognitive Engineering and Decision Making, 4(4), 289-303.

Sawyer, P. \& Kotonya, G. (2001). Software requirements. In P. Bourque \& R. Fairley (Ed.), Guide to the Software Engineering Body Knowledge (pp. 30-55). Washington, United States: IEEE Computer Society.

Schwendimann, B., Rodriguez-Triana, M., Vozniuk, A., Prieto, L., Boroujeni, M., Holzer, A. \& Dillenbourg, P. (2017). Perceiving learning at a glance: A systematic literature review of learning dashboard research. IEEE Transactions on Learning Technologies, 10(1), 30-41.

Smalheiser, N. R. (2017a). Chapter 9 - Null Hypothesis Statistical Testing and the t-Test Data Literacy (pp. 127-136): Academic Press.

Smalheiser, N. R. (2017b). Chapter 11 - ANOVA Data Literacy (pp. 149-155): Academic Press.

Sommerville, I. (2007). Software engineering. Harlow, England.

Thomas, J. \& Cook, K. (2006). A visual analytics agenda. IEEE Computer Graphics and Applications, 26(1), 10-13.

Verbert, K., Duval, E., Klerkx, J., Govaerts, S. \& Santos, J. (2013). Learning Analytics Dashboard Applications. American Behavioral Scientist, 57(10), 1500-1509.

Ware, C. (2004). Information visualization: Perception for design (2nd ed.). San Francisco, CA: Elsevier.

Wexler, S., Shaffer, J. \& Cotgreave, A. (2017). The big book of dashboards: visualizing your data using real-world business scenarios. Hoboken, NJ: Wiley.

Wolf, R. (2016). Dashboard design. Strategic Finance, 97(12), 20-27.

Zoya, H. \& Valeriy, S. (2016). Factor of competitiveness of universities under globalization of educational markets. USV Annals of Economics and Public Administration, 16, 106-110. 